\title{
Comportamiento del orificio de implantación del catéter en pacientes en diálisis peritoneal en relación a los cuidados
}

\author{
A. Concepción Gómez Castilla - J. Lucas Martín Espejo - Carmen Trujillo Campos - Ángela Laguillo de \\ Castro - Mercedes Tejuca Marenco - Encarnación López Bermúdez - Cristina Ortega Cuadro - Julia \\ Rodríguez Pérez - Piedad González de la Peña García - Flora López González
}

Grupo Andaluz de Diálisis Peritoneal - Hospitales: Virgen Macarena (Sevilla), Virgen del Rocío (Sevilla), Carlos Haya (Málaga), Puerta del Mar (Cádiz), Puerto Real (Cádiz), Reina Sofía (Córdoba), Virgen de las Nieves (Granada), San Cecilio (Granada), Jerez de la Frontera (Cádiz), Infantil V. del Rocío (Sevilla).

\section{Resumen}

Las infecciones del orificio de salida en diálisis peritoneal son la causa de morbilidad más frecuente en los pacientes sometidos a esta técnica. Existen numerosos procedimientos para los cuidados del orificio y no resulta fácil definir un único método que garantice el buen estado del orificio. Con el fin de valorar el comportamiento del orificio relacionado con el procedimiento utilizado en sus cuidados estudiamos a 306 pacientes durante 24 meses, recogiendo variables sociodemográficas y clínicas.

Hemos encontrado una alta incidencia de infecciones del orificio producidas por gérmenes gram positivos de piel y mucosas, con una correlación fuerte con el hecho de que el paciente/familiar cuidador sea portador nasal de estafilococo áureo y que aparecen con mayor frecuencia en los pacientes que no retiran el apósito para la ducha. Así mismo hemos detectado un aumento en infeccio-

Correspondencia:

Concepción Gómez Castilla

Unidad de Diálisis Peritoneal

Hospital Virgen Macarena

Av. Dr. Fedriani s/n

41071 Sevilla nes por pseudomona cuando el paciente no procede al secado del orificio con secador.

PALABRAS CLAVE:

- ORIFICIO DE SALIDA

- DIÁLISIS PERITONEAL

- PROCEDIMIENTO DE CUIDADOS

Behaviour of the catheter wound in peritoneal dialysis patients in relation to care

\section{Abstract}

Infections of the catheter wound in peritoneal dialysis are the most frequent cause of morbility in patients who undergo this technique. There are a number of procedures for the care of the wound and it is not easy to define a single method that will guarantee good condition of the wound. In order to evaluate the behaviour of the wound related to the procedure used in their care, we studied 306 patients over 24 months, compiling socio-demographic and clinical variables.

We found a high incidence of infections caused by gram-positive skin and mucous germs, with a strong correlation with the fact that the patient/family car 
er is a nasal carrier of staphylococcus aureus and that they appear more frequently in patients who do not remove the wound dressing in the shower. We also detected an increase in pseudomonas infections when the patient does not dry the wound with a hair-dryer.

\section{KEY WORDS}

- CATHETER WOUND

PERITONEAL DIALYSIS

- CARE PROCEDURE

\section{Introducción}

Las infecciones del orificio/tunel (IOT) de salida del catéter en diálisis peritoneal son la causa morbilidad mas frecuente actualmente en este tipo de pacientes ${ }^{1-4}$.

Estudios publicados hablan de un elevado índice de infecciones, que en ocasiones lleva a la perdida del catéter y/o peritonitis relacionada que, pueden ocasionar el deterioro de la membrana peritoneal y la pérdida de la capacidad dialítica de la misma ${ }^{1-3-4}$.

Los procedimientos para los cuidados del orificio son casi tan variados como los propios pacientes y no resulta fácil definir un único método que garantice el buen estado del orificio. Nosotros hemos querido observar cómo actuaban nuestros pacientes y cuál había sido el comportamiento del orificio, para lo que diseñamos este estudio descriptivo.

\section{Objetivos}

1. Valorar el comportamiento del orificio relacionado con el procedimiento utilizado en los cuidados del mismo.

2. Valorar las infecciones del orificio/túnel relacionado con determinadas variables contempladas en el estudio.

3. Valorar la incidencia de peritonitis relacionada con la infección del orificio.

\section{Pacientes y método}

- Estudio observacional descriptivo.
- Intervalo de estudio: enero 2004/diciembre 2005

- Población: Hemos estudiado 306 pacientes en programa de diálisis peritoneal en nuestra Comunidad Autónoma con una edad media de 53,6 \pm 20,3 , de los que $162(52,9 \%)$ eran hombres y 144 $(47,1 \%)$ eran mujeres, con un tiempo en diálisis de 22,3 \pm 19 , de ellos $138(45,1 \%)$ habían estado en DPAC y $168(54,9 \%)$ en DPA.

- Variables estudiadas: sexo, edad, tiempo en diálisis en meses, tipo de diálisis, dependencia del paciente, cuidador del orificio, estudio de portadores nasales de estafilococo áureo (SA) para paciente y familiar, frecuencia de ducha, ducha con apósito, procedimiento para la cura del orificio (limpieza, secado, protección y fijación), estado del orificio: tiempo que mantuvo el episodio de IOT, germen, época en que se produjo, evolución, peritonitis relacionada con IOT, peritonitis no relacionada, germen, hospitalización estancia y causa.

Los pacientes que se duchan sin apósito acostumbran a lavar el orificio y el catéter con agua y jabón utilizando para ello las manos limpias, pero después introducen en muchas ocasiones distintos procedimientos de desinfección; a la hora de definirlos en el estudio, solo hemos considerado el uso de agua y jabón cuando no utilizaban otro producto, y en adelante hemos considerado el más habitual, suero salino, solución desinfectante yodada, combinado cuando usaba varias soluciones desinfectantes, e incluimos en el apartado "otros", a los pacientes que usan habitualmente algún antibiótico en el orificio.

El nivel de dependencia fue valorado con el siguiente criterio:

- Pacientes autónomos: 0

- Pacientes autónomos para las actividades de la vida diaria, que necesitan o utilizan ayuda en la cura del orificio: 1

- Pacientes autónomos para las actividades de la vida diaria que necesitan o utilizan ayuda además de en la cura del orificio para la diálisis: 2

- Pacientes con autonomía en la deambulación que necesitan o utilizan ayuda para la higiene y/o el vestido, además de cura y diálisis: 3

- Pacientes dependientes además para otras actividades de la vida diaria, aunque tengan la capacidad de moverse solos o con ayuda de algún aparato: 4

- Pacientes totalmente dependientes: 5 
Consideramos portador habitual de estafilococo áureo (paciente/familiar que ha aparecido SA en frotis nasal en más de dos ocasiones en el intervalo del estudio) y portador ocasional (paciente/familiar que en frotis nasal se ha detectado SA en alguna ocasión y que desaparece en próximos controles sin haber efectuado tratamiento de descontaminación).

En la mayoría de nuestros pacientes, cuando se ha detectado el estafilococo áureo en frotis nasal, se efectúa tratamiento de descontaminación mediante mupirocina nasal según protocolo establecido en cada centro que puede variar levemente oscilando entre 5 días y 1 semana/ mes.

En la recogida de datos se definieron los orificios en razón a su aspecto: perfecto, costra, rojo (apariencia de eritema), granuloma, traumático y exudado, y posteriormente se agruparon como equívocos: los orificios con costra, los que presentaban eritema, los que tenían granuloma y los orificios traumáticos sin detección de germen. Los orificios traumáticos en los que se había detectado algún germen se consideraron infección, así como los orificios exudados.

Para definir las peritonitis, las consideramos relacionadas cuando el germen causante era el mismo que el detectado en el orificio y coincidente en el tiempo con el episodio de infección de dicho orificio, y no relacionada en los demás casos.

Para hospitalizaciones se valoró como relacionada únicamente las peritonitis fueran relacionadas o no con la infección del orificio y las relacionadas con perdida de catéter y hospitalización para implantación del siguiente.

No se contempló los días de hospitalización que pudieron producir la implantación del primer catéter al inicio de la entrada en programa, ni los ocasionados por trasplante por no considerarlos morbilidad. No relacionadas incluyó toda hospitalización no mencionada anteriormente.

El estudio estadístico de los datos se efectuó mediante programa SPSS 11.5.

\section{RESULTADOS}

Todos nuestros pacientes se secaban con gasa estéril y ninguno de ellos dijo utilizar toalla para el secado del orificio.

En la figura 1 observamos la evolución del orificio durante el estudio para las tres observaciones que hubo que controlar. Los episodios controlados son contabilizados en el momento en que se produce la alteración y no tiene relación en el tiempo, por lo que no suponen un punto de corte estructurado.



Figura 1. Evolución del orificio durante el estudio (24 meses)

La figura 2 representa el porcentaje de episodios infecciosos que se produjeron entre mayo y septiembre (verano) donde la temperatura es más alta en nuestro entorno y algunos de nuestros pacientes comienzan a utilizar ya el baño en piscinas o playa, observando una menor incidencia que durante el invierno, aunque no significativa.

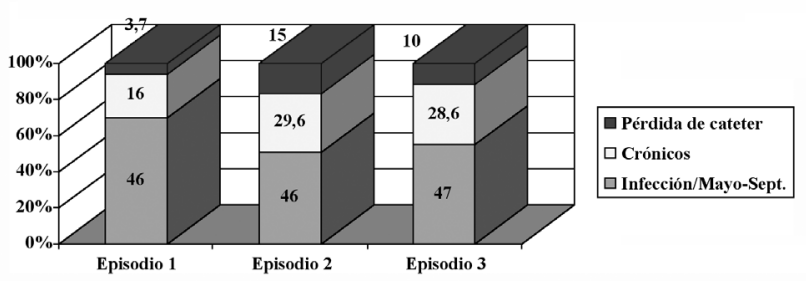

Figura 2. Duración del episodio infeccioso, época en que se produce y evolución a perdida de cateter

En la tabla 1 se observa como los mas bajos índices de infección se presentan en los pacientes que solo utilizan agua y jabón así como en los que utilizan povidona yodada. Hemos detectado asociación con $\mathrm{p}<$ 0,05 mediante chi-cuadrado para todas las opciones de limpieza del orificio excepto para el apartado otros. 
[ AC. Gómez Castilla, et al ]

Comportamiento del orificio de implantación del catéter en pacientes en diálisis peritoneal en relación a los cuidados

\begin{tabular}{|c|c|c|c|c|c|c|c|}
\hline \multirow{2}{*}{$\begin{array}{l}\text { Limpieza orificio } \\
\text { Agua y jabón }\end{array}$} & \multirow{2}{*}{$\begin{array}{l}\text { Estado orificio } \\
\text { Perfecto }\end{array}$} & \multicolumn{2}{|c|}{ Episodio 1} & \multicolumn{2}{|c|}{ Episodio 2} & \multicolumn{2}{|c|}{ Episodio 3} \\
\hline & & 24 & $80.0 \%$ & 27 & $90.0 \%$ & 30 & $100 \%$ \\
\hline & Equivoco & 5 & $16.7 \%$ & 2 & $6.7 \%$ & 0 & 0 \\
\hline & Infección & 1 & $3.3 \%$ & 1 & $3.3 \%$ & 0 & 0 \\
\hline \multirow[t]{3}{*}{ Suero salino } & Perfecto & 37 & $36.6 \%$ & 78 & $77.2 \%$ & 92 & $91.1 \%$ \\
\hline & Equivoco & 46 & $45.5 \%$ & 9 & $8.9 \%$ & 5 & $5.0 \%$ \\
\hline & Infección & 18 & $17.8 \%$ & 14 & $13.9 \%$ & 4 & $4.0 \%$ \\
\hline \multirow[t]{3}{*}{ Povidona yodada } & Perfecto & 48 & $56.5 \%$ & 81 & $95.3 \%$ & 83 & $97.6 \%$ \\
\hline & Equivoco & 31 & $36.5 \%$ & 2 & $2.4 \%$ & 2 & $2.4 \%$ \\
\hline & Infección & 6 & $7.1 \%$ & 2 & $2.4 \%$ & 0 & 0 \\
\hline \multirow[t]{3}{*}{ Combinado } & Perfecto & 26 & $31.7 \%$ & 61 & $74.4 \%$ & 77 & $93.9 \%$ \\
\hline & Equivoco & 38 & $46.3 \%$ & 9 & $11.0 \%$ & 1 & $1.2 \%$ \\
\hline & Infección & 18 & $22.0 \%$ & 12 & $14.6 \%$ & 4 & $4.9 \%$ \\
\hline \multirow[t]{3}{*}{ Otros } & Perfecto & 0 & 0 & 5 & $62.5 \%$ & 6 & $75.0 \%$ \\
\hline & Equivoco & 4 & $50.0 \%$ & 2 & $25.0 \%$ & 1 & $12.5 \%$ \\
\hline & Infección & 4 & $50.0 \%$ & 1 & $12.5 \%$ & 1 & $12.5 \%$ \\
\hline
\end{tabular}

Tabla 1. Distribución de la infecciones del orificio según el procedimiento utilizado

En la tabla 2 observamos que el mayor índice de infección se produce tanto en los pacientes portadores habituales como en los portadores ocasionales de estafilococo áureo. Siendo además los que más episodios de infección del orificio padecen a lo largo de los 24 meses de nuestro estudio, apareciendo también asociación con $p<0,05$ mediante chi-cuadrado.

\begin{tabular}{|c|c|c|c|c|c|c|c|}
\hline \multirow{2}{*}{$\begin{array}{l}\text { Estafilococo } \\
\text { áureo nasal } \\
\text { Portador habitual }\end{array}$} & \multirow{2}{*}{$\begin{array}{l}\text { Estado orificio } \\
\text { Perfecto }\end{array}$} & \multicolumn{2}{|c|}{ Episodio 1} & \multicolumn{2}{|c|}{ Episodio 2} & \multicolumn{2}{|c|}{ Episodio 3} \\
\hline & & 5 & $20.0 \%$ & 18 & $72.0 \%$ & 23 & $92.0 \%$ \\
\hline & Equivoco & 10 & $20.0 \%$ & 3 & $12.0 \%$ & 0 & 0 \\
\hline & Infección & 10 & $40.0 \%$ & 4 & $16.0 \%$ & 2 & $8.0 \%$ \\
\hline \multirow[t]{3}{*}{ Portador ocasional } & Perfecto & 9 & $28.1 \%$ & 23 & $71.9 \%$ & 28 & $87.5 \%$ \\
\hline & Equivoco & 12 & $37.5 \%$ & 4 & $12.5 \%$ & 1 & $3.1 \%$ \\
\hline & Infección & 11 & $34.4 \%$ & 5 & $15.6 \%$ & 3 & $9.4 \%$ \\
\hline \multirow[t]{3}{*}{ No portador } & Perfecto & 85 & $45.5 \%$ & 152 & $81.3 \%$ & 176 & $94.1 \%$ \\
\hline & Equivoco & 81 & $43.3 \%$ & 16 & $8.6 \%$ & 7 & $3.7 \%$ \\
\hline & Infección & 21 & $11.2 \%$ & 19 & $10.2 \%$ & 4 & 2.1 \\
\hline \multirow[t]{3}{*}{ No conocido } & Perfecto & 36 & $58.1 \%$ & 59 & $95.2 \%$ & 61 & $98.4 \%$ \\
\hline & Equivoco & 21 & $33.9 \%$ & 1 & $1.6 \%$ & 1 & $1.6 \%$ \\
\hline & Infección & 5 & $8.1 \%$ & 2 & $3.2 \%$ & 0 & $4.9 \%$ \\
\hline
\end{tabular}

Tabla 2. Distribución de la infecciones del orificio en portadores nasales de estafilococo áureo 
En la figura 3 observamos el comportamiento del orificio según quién efectúa los cuidados, destacando el alto índice de orificios infectados cuando el cuidador que efectúa los cuidados es el familiar del paciente.

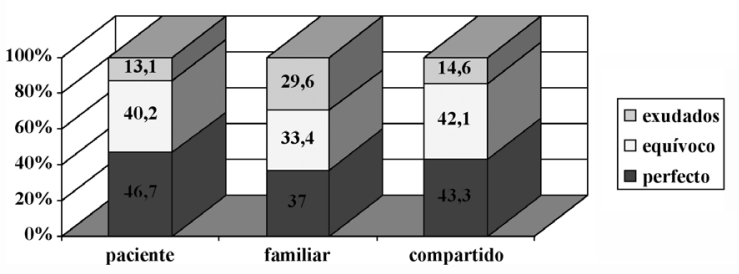

Figura 3. Comportamiento del orificio $\mathrm{r} / \mathrm{c}$ el cuidador

En la figura 4 observamos como el mayor índice de infecciones del orificio se presenta en los pacientes que necesitan ayuda para caminar y en los que nece- sitan ayuda en la higiene y el vestido. Hemos observado que estas diferencias se relacionan más con el tipo de cuidador del orificio que con la frecuencia de ducha.

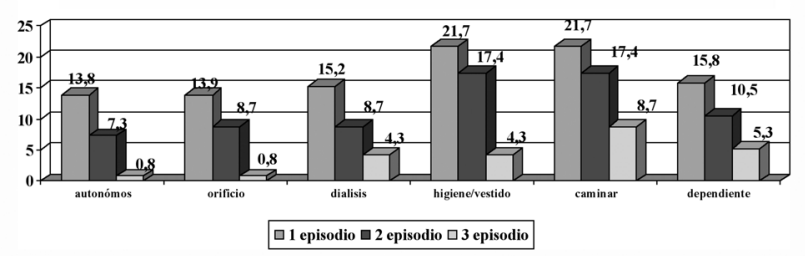

Figura 4. Infecciones del orificio r/c nivel de dependencia/tipo de ayuda que recibe el paciente

La tabla 3 refleja la distribución de gérmenes como causantes de las infecciones del orificio, destacando el alto porcentaje de infecciones producidas por gérmenes gram positivo cuyo hábitat es piel y mucosas.

\begin{tabular}{lrrrrrrrrr}
\multicolumn{1}{c}{ Gérmen causante } & \multicolumn{3}{c}{ Episodio 1 } & \multicolumn{3}{c}{ Episodio 2 } & \multicolumn{3}{c}{ Episodio 3 } \\
& Frec & $\%$ & válido & Frec & $\%$ & válido & Frec & $\%$ & válido \\
Gram pos. piel y mucosas & 44 & 14.3 & 65.7 & 18 & 5.9 & 52.9 & 8 & 2.6 & 66.7 \\
Gram pos. orofaringe & 1 & 0.3 & 1.5 & 0 & & & 0 & & \\
Gram pos. intestinal & 0 & & & 1 & 0.3 & 2.9 & 1 & 0.3 & 8.3 \\
Gram neg. ambiental & 10 & 3.3 & 14.9 & 5 & 1.6 & 14.7 & 2 & 0.7 & 16.7 \\
Gram neg. intestinal & 12 & 3.9 & 17.9 & 9 & 2.9 & 26.5 & 1 & 0.3 & 8.3 \\
Hongos & 0 & & & 1 & 0.3 & 2.9 & & & \\
Total & 67 & & & 34 & 11.1 & 100.0 & 12 & 3.9 & 100.0
\end{tabular}

Tabla 3. Distribución de los gérmenes en el orificio

En la figura 5 observamos la incidencia de infecciones del orificio relacionado con la frecuencia de ducha, destacando el alto índice de infecciones detectadas en el primer episodio de los pacientes que se duchan una vez a la semana. Sin embargo al efectuar chi cuadrado no detecta asociación de estas variables.

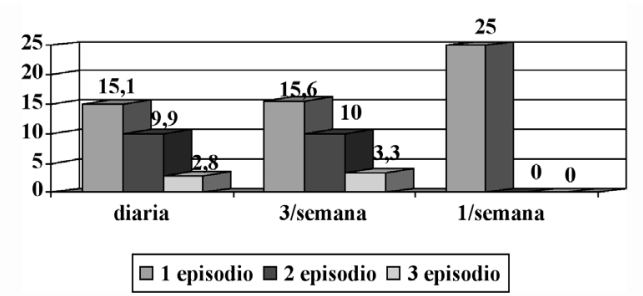

Figura 5. Infecciones del orificio r/cfrecuencia de ducha
En la figura 6 observamos la distribución de infecciones del orificio por pseudomonas en los pacientes que utilizan secador para el secado del orificio y los que no lo utilizan.

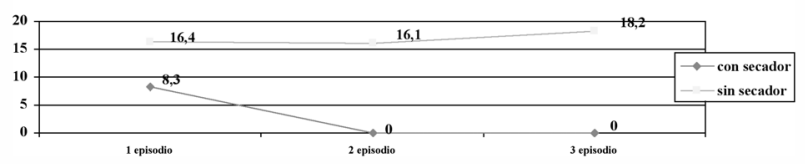

Figura 6. Infecciones del orificio por pseudomonas r/c el uso de secador

En la figura 7 presentamos la distribución de gérmenes que aparecen en el orificio cuando los pacientes se duchan con apósito y cuando éste es retirado para la ducha, destacando el porcentaje más elevado 
de la mayoría de los gérmenes cuando el paciente no retira el apósito para ducharse, sino que se ducha con él y procede luego a la cura, observándose diferencias significativas.

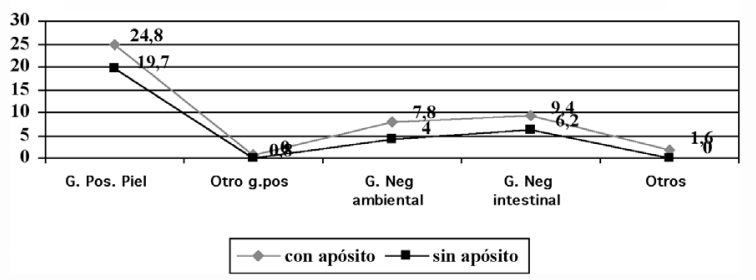

Figura 7. Distribución de gérmenes según utilizacion de apósito o no durante la ducha

En la figura 8 representamos el porcentaje de pacientes que ha sido hospitalizado por causa relacionada 0 no relacionada con peritonitis o problemas infecciosos del catéter y los días de estancia media que tuvieron en total.

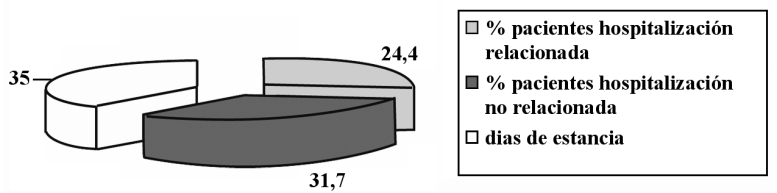

Figura 8. Morbilidad traducida en hospitalizaciones del paciente y relación con peritonitis o problemas infecciosos del cateter

En la figura 9 observamos el porcentaje de pacientes que padece peritonitis en los distintos episodios observados en los 24 meses del estudio, distinguiendo la incidencia de peritonitis relacionadas con las infecciones del orificio y las no relacionadas. Presentando una media de 0,16 $\pm 0,48$ episodios/paciente para las peritonitis relacionadas y $0,23 \pm$ 0,52 episodios/paciente para la peritonitis no relacionadas.

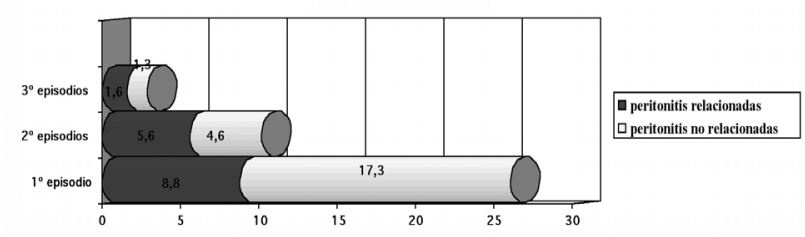

Figura 9. Incidencia de peritonitis

En la tabla 4 observamos la elevada incidencia de gérmenes gram positivo de piel y mucosas como causante de las peritonitis.

Podemos concluir que en nuestro estudio hemos encontrado una alta incidencia de infecciones del orificio producidas por gérmenes gram positivo de piel y mucosas con una correlación fuerte con el hecho de que el paciente/familiar cuidador sea portador nasal de estafilococo áureo y que aparecen con mayor frecuencia en los pacientes que no retiran el apósito para la ducha.

\begin{tabular}{lrrrrrrrrrr}
\multicolumn{1}{c}{ Gérmen causante } & \multicolumn{3}{c}{ Episodio 1 } & \multicolumn{3}{c}{ Episodio 2 } & \multicolumn{3}{c}{ Episodio 3 } \\
& Frec & $\%$ & válido & Frec. & $\%$ & válido & Frec. & $\%$ & válido \\
Gram pos. piel y mucosas & 32 & 10.5 & 53.3 & 14 & 4.6 & 51.8 & 4 & 1.4 & 50.0 \\
Gram pos. orofaringe & 6 & 2.0 & 10.0 & 1 & 0.3 & 3.7 & 0 & & \\
Gram pos. intestinal & 3 & 1.0 & 5.0 & 1 & 0.3 & 3.7 & 0 & & \\
Gram neg. piel & 1 & 0.3 & 1.7 & 0 & & & 0 & & \\
Gram neg. respiratorio & 1 & 0.3 & 1.7 & 0 & & & 0 & & \\
Gram neg. ambiental & 5 & 1.6 & 8.3 & 2 & 0.7 & 7.4 & 3 & 1.0 & 37.5 \\
Gram neg. intestinal & 3 & 1.0 & 5.0 & 5 & 1.6 & 18.5 & 0 & & \\
Hongos & 2 & 0.7 & 3.3 & 1 & 0.3 & 3.7 & 1 & 0.3 & 12.5 \\
Otros & 0 & & & 3 & 1.0 & 11.1 & 0 & & \\
Total & 60 & 19.6 & 100 & 27 & 8.8 & 100 & 8 & 2.6 & 100.0
\end{tabular}


Así mismo hemos detectado un aumento en infecciones por pseudomona cuando el paciente no procede al secado del orificio con secador, lo que puede contribuir al mantenimiento de la humedad.

Las diferencias que existen en las infecciones del orificio según el procedimiento de limpieza creemos está justificado por la dinamicidad del proceso y que es más efecto de las infecciones que causa de las mismas.

Es importante la incidencia de peritonitis que pueden estar relacionadas con las infecciones del orificio así como la morbilidad en días de hospitalización que produce, y la necesidad del cambio de catéter sobre todo en episodios crónicos y repetitivos, produciéndose sobre todo en gérmenes como estafilococo áureo y pseudomonas.

\section{Bibliografia}

1. Coronel $\mathrm{F}$ et al. Manual práctico de diálisis peritoneal. Atrium; 2005.

2. Muñoz Poyato J. Manual de protocolos y procedimientos de actuación de enfermería nefrológica SEDEN. Madrid: Entheos; 2001.

3. Andreu L, Force E. "500 cuestiones que plantea el cuidado del paciente renal". $2^{\mathrm{a}}$ ed. Barcelona: Masson; 2001.

4. Gómez C, Sánchez J. Portadores nasales de estafilococo áureo en diálisis peritoneal. Las infecciones del orificio. En: Libro de comunicaciones del XXVII Congreso de la Sociedad Española de Enfermería Nefrológica; Bilbao octubre 2002. Barcelona: HOSPAL; 2002. 